\title{
3 WINS Fitness: Sustainable, Affordable, and Scalable Fitness Program for the Next Generation
}

\author{
Steven Loy
}

Today, students and faculty in kinesiology have an opportunity to change the health trajectory of millions in the United States, if not the world. While extremely confident we have the tools, I say this conservatively because it requires us to take control of our destiny. Only 16 years ago, I experienced the reality that despite a 1990s system-wide name change of hundreds of universities to include the kinesiology major, many of my esteemed public health colleagues in California did not know what kinesiology was, and thus, there were few jobs in the public health sector that required an "exercise professional." In 2019, given the majority of our students' parents are uninformed of what the kinesiology major represents, then, presumably, the public at large is also oblivious to the education provided to our students. We have done a remarkably poor job of marketing who we are, and we must do better so that our public health colleagues and the public sector look to us for healthier life solutions.

Physical inactivity is the fourth leading cause of death worldwide, with direct health care-related costs in the hundreds of billions of dollars, in addition to monies associated with many other diseases whose risk increases with physical inactivity. But this paper is not about providing a detailed elucidation of the problem, it is about providing a pragmatic solution. Through the course of my career, I have seen exercise evolve from a mere correlation with better health and longevity to the recognition of a robust cause-and-effect relationship. What a boon to our profession! Our value as promotors of exercise has been validated! We can translate this into kinesiology discipline-specific health careers! We can make a difference!

However, as a college professor, I found myself frustrated with the knowledge that we could make a difference for people, and yet, professionally, what we were doing was largely making recommendations. More recently, I have read papers outlining what programs must do to be successful, and yet, the majority of the programs were funded research interventions that did not last beyond the dates of the research, or they just said what to do without actually creating a program. We have experienced an explosion of kinesiology majors across the country, in part driven by students using kinesiology as a prerequisite for allied health careers, such as physical therapy, occupational therapy, nursing, etc, and not for the inherent human movement we research and expound. At California State University, Northridge, we recently peaked at 2400 majors, and with that was additional frustration caused by the vast number of students asking me, "what else can I do with my kinesiology major?"

And so, I challenged my classes to take their knowledge to the people and make changes in community health by increasing

The author is with the Department of Kinesiology, California State University, Northridge, Northridge, CA. Loy (steven.loy@csun.edu) is corresponding author. people's physical activity. Show and educate participants, particularly in underserved communities where health disparities are the greatest, what to do to meet the Centers for Disease Control and Prevention guidelines for physical activity and, more importantly, encourage them by being present beyond the usual 12-week research protocol. I found our Millennials and Generation $\mathrm{Z}$ students unwilling/unprepared/unable to take on this challenge. Thus, in 2011, I created the 100 Citizens program, now known as 3 WINS Fitness (www.3winsfitness.com), based on the premise of involving students so they could understand the power they had to make world-altering change.

The research is definitive. A program of community physical activity must be sustainable, accessible, and scalable. With this in mind, I decided NOT to seek external funding to run the program and, instead, concomitantly empower students, who receive academic internship credit or who volunteer, to deliver a FREE exercise program to underserved communities. At present, we have conducted programs in public parks, faith-based institutions, and public schools, and we currently conduct 6 programs serving more than 400 participants throughout the year. During the past 8 years, we have scaled the program to other California state universities, though their programs are not as large as ours (that will be for a later evolution), and we have developed our program to serve 4 different fitness levels, including a falls prevention group, and deliver a free diabetes program modified from the National Diabetes Prevention Program. The 3 WINS Fitness program is sustainable, affordable, replicable, and scalable.

The problem of physical inactivity threatens to overwhelm us. Because we offer our program in the morning after school begins, we reach the very group that is seen as at a higher risk for obesity, African American and Hispanic females, who can also have great influence on childhood obesity through role modeling and offering healthier nutrition in the home. Their stories are remarkable. Capitalizing on rising kinesiology student numbers only strengthens the potential outreach of the program, and the community family-like bond formed among students and participants is a factor in our success. Over 8 years, the numbers of students who attribute their 3 WINS Fitness experience with their job acquisition and career satisfaction continue to grow. These are our 3 WINS: student professional development, participant health, and community health.

The fact that 3 WINS Fitness is FREE without external funding for sustainability makes scaling the program even more compelling. Our program can be replicated in more than 700 universities, but scaling across the country will cost money. We will be seeking funding to create a "train the trainer model," that is, 1 university in each state will receive intensive training at California State University, Northridge to teach the remaining universities in their state. Seldom is there an opportunity to make change on the scale we are discussing. This is the time. Kinesiology departments can lead the way, and 3 WINS Fitness could become even more robust when combined with public health 
and nutrition students. We have thousands of students receiving their education who can make a difference while in school and be followed by thousands more year after year. We have proven sustainability and capacity. This generation of Millennials and
Generation $\mathrm{Z}$ students can control their destiny and teach millions of people to do the same. The question is not, can this be done? But, when can we start? Large-scale problems require INNOVATIVE solutions, and it is time to address physical inactivity. 